\title{
Extracellular vesicles and infectious diseases: new complexity to an old story
}

\author{
Jeffrey S. Schorey' and Clifford V. Harding ${ }^{2}$ \\ 'Department of Biological Sciences, Eck Institute for Global Health, University of Notre Dame, Notre Dame, Indiana, USA. ²Department of Pathology, Case Western Reserve University, \\ University Hospitals Case Medical Center, Cleveland, Ohio, USA.
}

\begin{abstract}
Exosomes and other extracellular microvesicles (ExMVs) have important functions in intercellular communication and regulation. During the course of infection, these vesicles can convey pathogen molecules that serve as antigens or agonists of innate immune receptors to induce host defense and immunity, or that serve as regulators of host defense and mediators of immune evasion. These molecules may include proteins, nucleic acids, lipids, and carbohydrates. Pathogen molecules may be disseminated by incorporation into vesicles that are created and shed by host cells, or they may be incorporated into vesicles shed from microbial cells. Involvement of ExMVs in the induction of immunity and host defense is widespread among many pathogens, whereas their involvement in immune evasion mechanisms is prominent among pathogens that establish chronic infection and is found in some that cause acute infection. Because of their immunogenicity and enrichment of pathogen molecules, exosomes may also have potential in vaccine preparations and as diagnostic markers. Additionally, the ability of exosomes to deliver molecules to recipient cells raises the possibility of their use for drug/therapy delivery. Thus, ExMVs play a major role in the pathogenesis of infection and provide exciting potential for the development of novel diagnostic and therapeutic approaches.
\end{abstract}

\section{Introduction}

Cell-cell communication is critical for maintaining body homeostasis and responding quickly and appropriately to cellular damage and pathogen invasion. This communication can be mediated by direct cell-cell contact, but is often directed by soluble factors such as hormones, cytokines, and inflammatory mediators that can communicate to a large number of responding cells either locally or at dispersed sites. Recently, extracellular microvesicles (ExMVs) have also been found to facilitate cell-cell communication. Because ExMVs are released from most nucleated cells, and because their composition changes under different physiological and pathological conditions, they are increasingly recognized as important regulators of cellular functions and disease mechanisms. This Review will discuss recent studies of the composition and function of ExMVs during viral, bacterial, parasitic, and fungal infections as well as their role in the discrimination of infectious prions; in these settings, ExMVs may contribute to either host defense or pathogen immune evasion mechanisms.

The ExMVs released from cells during an infection can be either pathogen- or host-derived. The former includes outer membrane vesicles (OMVs) from gram-negative bacteria and membrane vesicles from gram-positive bacteria and mycobacteria (1, 2). The function and content of these bacteria-generated vesicles has been excellently reviewed elsewhere (3-5). Parasitic and fungal pathogens also release ExMVs, which may function in modulating

Conflict of interest: The authors have declared that no conflict of interest exists. Reference information: J Clin Invest. 2016;126(4):1181-1189. doi:10.1172/JCI81132. the immune response (6-8). ExMVs from host cells are divided into three main categories: apoptotic bodies, exosomes, and microvesicles. All three of these cell-derived vesicles are enclosed by a lipid bilayer, but vary in size (from $30 \mathrm{~nm}$ to $2,000 \mathrm{~nm}$ in diameter) as well as in composition and mechanism of biosynthesis (9).

The unique features of ExMVs in cell-cell communication stem from their complex composition, which allows for a finely tuned control over the communication process and the potential engagement of multiple receptors and signaling pathways in responding cells. Further, the presence of signaling lipids, proteins, and various species of RNA within a single structure can lead to a rapid and profound change in the target cell, enabling a rapid response to cellular perturbations. The responding cell can in turn release molecules, which can act locally or systemically. These mechanisms may be induced under physiological or pathological conditions. Although the complexity of exosomes broadens their functional impact, it makes the study of their activity difficult, as the effect of the vesicles is a result of all the different components within them, including lipids, proteins, carbohydrates, and RNA. Moreover, ExMVs produced during the course of an infection will be diverse in activity and composition, as they are derived from multiple cell sources (potentially including vesicles derived from both host and pathogen). This makes defining their function challenging, as the different ExMV populations may have synergistic or even opposing effects on recipient cells.

Of the different ExMVs, exosomes have been the most studied in the context of infection. However, the purity of vesicles in various studies was not always defined, and the vesicle populations may have contained both exosomes and microvesicles, which 
overlap in size and density. Nevertheless, we will use the terminology as defined in the original studies when discussing the results. The observed composition and function of the ExMVs may also be affected by the animal model used, the experimental design, the cell types chosen for the infection and source of vesicles, and the type of recipient cells, among other factors.

\section{Immune responses to extracellular versus intracellular pathogens}

Pathogens can reside intracellularly or extracellularly, and compartmental lifestyle is a major factor in dictating what evasion mechanisms are used by the pathogen and what types of host immune responses are protective. The pathogen's lifestyle is also relevant to the role of ExMVs in disease mechanisms. For pathogens that are primarily extracellular, pathogen-associated molecular patterns (PAMPs) that are shed or secreted either via membrane vesicles or as soluble factors can directly interact with pattern recognition receptors (PRRs) on the plasma membrane of host cells. Alternatively, PAMPs or antigens may be endocytosed by antigen-presenting cells (APCs), allowing interaction with intracellular receptors or processing of antigens for presentation by MHC class II molecules. There are analogous mechanisms for intravacuolar pathogens, which communicate by endocytosis and exocytosis with the extracellular space. For example, macrophages infected by Mycobacterium tuberculosis may release bacterial molecules either via membrane vesicles derived from intraphagosomal bacteria (10) or by incorporation of bacterial molecules into host membranes or intraluminal vesicles (ILVs) for release through ExMVs $(11,12)$. In contrast, cytosolic pathogens penetrate through membranes to reach the cytosol (or nucleus), and their PAMPs may interact with cytosolic PRRs. The ability of cytoplasmic pathogens to release molecules into the extracellular space is unclear but may occur by lysis of the infected cell or release of ExMVs that include cytosol-derived materials. In this Review, we will discuss the biogenesis, composition, and function of ExMVs, including exosomes, during viral, bacterial, or parasitic infections and how the functions of these vesicles may benefit the host or pathogen.

\section{Exosome production}

Turnover of plasma membrane receptors includes endocytosis and trafficking to multivesicular bodies (MVBs), which can subsequently fuse with lysosomes to mediate protein degradation (13-15). However, a subpopulation of MVBs can also fuse with the plasma membrane, resulting in release of the ILVs as exosomes $(16,17)$. The mechanism for MVB biogenesis and exosome release is still being defined, but several models have been suggested as a mechanism for ILV formation (reviewed in ref. 18).

Not surprisingly, pathogens have been shown to hijack the host exosome biosynthetic machinery as part of their survival strategy. Viruses such as paramyxoviruses, hepatitis $\mathrm{C}$ virus (HCV), rhabdoviruses, filoviruses, herpesviruses, and hepatitis B virus (HBV) have been shown to utilize or require the endosomal-sorting complexes required for transport (ESCRT) pathway to promote their release (reviewed in ref. 19). Chlamydia trachomatis was recently shown to use MVBs as a source of sphingolipids, phospholipids, and cholesterol, and recruitment of MVBs to the chlamydial inclu- sion was dependent on the late endocytic GTPase Rab39a (20). Moreover, pathogens have been shown to modulate the exosome biosynthetic pathway by affecting recruitment of host factors to MVBs and ILVs. For example, the human parainfluenza virus type 1 recruits the ESCRT proteins ALIX and CHMP4B, limiting their availability for exosome biogenesis (21). Some intracellular bacterial pathogens, including $M$. tuberculosis, are also known to interfere with the host proteins, such as ESCRT proteins, implicated in exosome biogenesis (22).

\section{Exosome composition}

Exosomes contain many types of biomolecules, including proteins, carbohydrates, lipids and nucleic acids. Exosome composition is dynamic and varies depending on the cell's origin, its physiological and pathological state, and even the cellular release site (23). Exosome protein composition can also mark the existence of disease pathology such as cancers or inflammatory diseases (24); however, exosomes also contain a number of common proteins as well as proteins that participate in vesicle formation and trafficking $(25,26)$. Exosome lipid composition has also been well characterized (27). A number of reviews have highlighted the protein and lipid content of exosomes $(28,29)$, and various databases, including ExoCarta (http://www.exocarta.org/) and Vesiclepedia (http://microvesicles.org/), have cataloged the protein, lipid, and RNA content of exosomes.

In addition to host factors, a number of pathogen-derived components have been found on exosomes after infection, but the mechanisms by which these diverse pathogen-derived components are sorted to MVBs and into exosomes remain poorly defined. Our current understanding stems primarily from viral studies, which have shown that viral assembly and exosome biogenesis share many commonalities (19). These similarities and the presence of viral proteins in exosomes suggest a degree of crosstalk as a mechanism for sorting the viral proteins into exosomes; however, some viral proteins such as the HIV protein Nef may contain signals to mediate their direct sorting into exosomes $(30,31)$. Even less is known about the incorporation of nonviral components into exosomes. Studies of mycobacteria suggest that a subset of mycobacterial proteins can be trafficked to exosomes independently of how they gained access to host macrophages. Proteins taken in both as free proteins via endocytosis or as part of the mycobacteria via phagocytosis were trafficked to exosomes (32), suggesting that mycobacterial proteins contain signals directing them to the MVB during exosome biogenesis. Moreover, recent evidence indicates that trafficking of mycobacterial proteins to exosomes is dependent on host E3 ligasemediated ubiquitination (33). The importance of ubiquitination for trafficking of other pathogen-derived proteins is unknown, but given the presence of E1, E2, and E3 ligases in both the cytoplasm and endosomal compartments, it is likely that this mechanism may function in the context of other pathogens that reside in these compartments.

A significant area of ongoing research stems from pioneering work done by Ratajczak et al. (34) and Valadi et al. (35), who independently showed exosome-mediated transfer of mRNA and $\mathrm{miR}$ to recipient cells. Recent studies have sought to define the types and sequence of the packaged RNA, their ability to be 
A
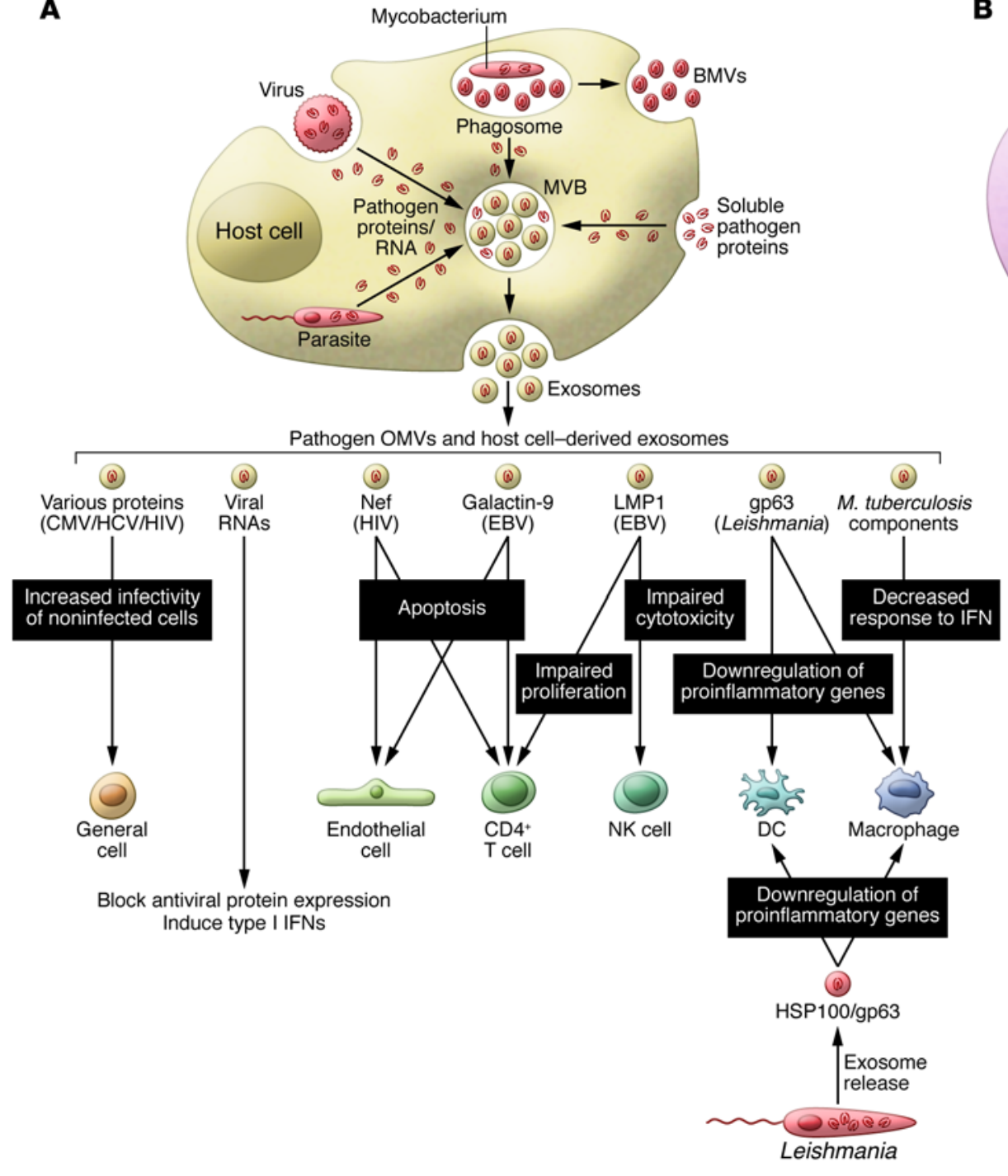

B

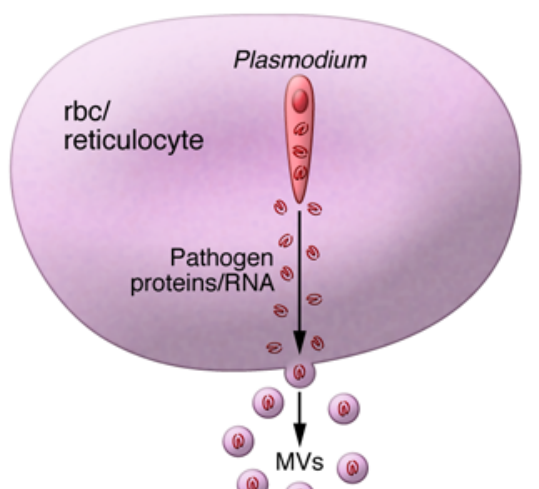

(1) MVs

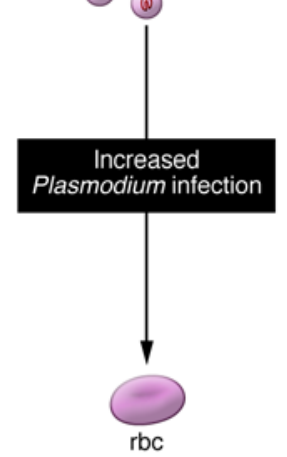

Figure 1. Inhibition of host immunity by exosomes and other ExMVs during the course of an infection. (A) Virus-, parasite-, and bacteria-infected cells release components that can be trafficked to MVBs and released on exosomes or the pathogens themselves release exosomes or microvesicles (MVs) and BMVs. These exosomes and other extracellular vesicles can inhibit an immune response and likely do so through multiple mechanisms acting on multiple cells. Examples include exosomes containing microbial molecules such as HIV Nef or Leishmania gp63, which can block T cell activation or induce apoptosis of immune effector cells. Exosomes from M. tuberculosis- or Leishmania-infected macrophages or from Leishmania itself can also limit the proinflammatory response in target macrophages and DCs. In contrast, exosomes from CMV-, HIV-, and HCV-infected cells can enhance the susceptibility of noninfected cells. (B) Similar results were seen with microvesicles from Plasmodium-infected rbc.

transferred between cells, their function once transferred, and the mechanism by which they are trafficked to MVBs and into exosomes. Importantly, the RNA present within exosomes is biologically active, indicating that the RNA can function to modulate the protein profile and cellular state of the recipient cell (reviewed in ref. 36). However, exosomal RNA content is dictated by its cellular origin and the physiological state of the cell (37), indicating that the incorporation of RNA into vesicles is a regulated event leading to selective packaging of RNA into exosomes and other ExMVs $(38,39)$.

In addition to host RNA, there is clear evidence for incorporation of viral RNAs into exosomes. The presence of HCV viral RNA in exosomes was found to be dependent on the ESCRT machinery and annexin A2, an RNA-binding protein involved in membrane vesicle trafficking (40). Similarly, the ESCRT II subunit EAP30 forms a complex with HIV Gag and staufen 1 to control HIV-1 RNA trafficking and gene expression (41). The underlying mechanism by which EAP3O facilitates HIV-1 genomic RNA trafficking remains unclear, although EAP30 may constitute part of the ribonucleoprotein complex that mediates the nuclear export of viral RNA. Unspliced HIV-1 RNA species are also recruited to exosomes, and this recruitment is dependent on the $5^{\prime}$ end of the $\mathrm{Gag}$ p17 ORF (42). Interestingly, we have found mycobacterial RNA within exosomes released from $M$. tuberculosis-infected macrophages (43), suggesting that the sorting of pathogen RNA to exosomes may be a more general phenomenon of host cell infection. In most cases, the function of the pathogen-derived RNA within exosomes remains to be defined. 


\section{Modulation of the immune response by ExMVs during an infection}

A fundamental question is whether the functions of ExMVs bearing pathogen-derived molecules drive host defense and pathogen elimination or mediate the dissemination of virulence factors to promote pathogen survival and disease. In some cases, both of these may occur simultaneously and in balance. This is a complex question that depends on both host and pathogen factors as well as environmental factors. The answer to this question for various pathogens has been elusive, as we lack many of the tools needed for investigation, e.g., robust methods to specifically inhibit production of exosomes or other ExMVs in order to evaluate their roles in immune responses and infection control. Nevertheless, studies using various in vitro and in vivo infection models have provided some insight into the function of exosomes and other ExMVs in host defense and immune evasion. A commonality observed for many in vivo infection studies is an elevated concentration of blood ExMVs, again supporting a functional relevance for these vesicles during an infection (44-46).

Contributions of extracellular vesicles to immune evasion. Exosomes and other ExMVs have been implicated in the pathogenesis of many different viruses (Figure 1). Upon release, these vesicles are "captured" by cells, and the transfer of host and viral proteins and/ or RNA could enhance viral infection and replication in recipient cells, or inhibit the immune response through induction of apoptosis or by blocking key cellular responses. In the case of human CMV, microvesicles released by infected cells contain soluble DC-SIGN, a C-type lectin family molecule, in complex with CMV glycoprotein B. Release of this complex through microvesicles and its interaction with target cells appears to increase the susceptibility of recipient cells to CMV infection (47). Similarly, in HCVinfected patients, the interaction of the cellular membrane protein CD81 with HCV envelope glycoprotein E2 and the release of this complex within microvesicles and subsequent interaction with recipient cells increase the susceptibility of recipient cells to HCV (48). Exosomes and other ExMVs released from HIV-1-infected peripheral blood mononuclear cells (PBMCs) or from megakaryocytes and platelets contain CCR5 and CXCR4, respectively. In both cases, the transfer of these chemokine receptors to target cells enhances their susceptibility to HIV infection $(49,50)$. Work by Campbell and colleagues showed that Nef is present in exosomes secreted from transfected HEK 293 cells, which, upon fusion with uninfected Jurkat T cells, restore infectivity to Nef-negative HIV virions (31). Together, these results suggest that ExMVs released during viral infections enhance the infectivity of neighboring cells, preparing the way for the soon-to-be-released viral particles.

Induction of apoptosis or functional suppression of immune cells appears to be another general role for ExMVs during a viral infection. For example, Nef-containing exosomes released from transfected SupT1 and Jurkat T cells can induce CD4 $4^{+} \mathrm{T}$ cell apoptosis in vitro, pointing to the possible role of these exosomes in the T cell depletion inherent to HIV pathogenesis (30). Similar results were observed for endothelial cells exposed to Nef (51). Galectin 9, which is present in exosomes released from EBV-infected cells, can interact with the $\mathrm{T}$ cell $\mathrm{Ig}$ mucin 3 and induce apoptosis of EBV-specific CD4 ${ }^{+} \mathrm{T}$ cells (52). Dukers and colleagues discovered that latent membrane protein 1 (LMP1), a signal transduction pro- tein important in EBV infection, blocked the proliferation of T cells and inhibited NK cell cytotoxicity (53). LMP1 is present on exosomes from EBV-infected cells, suggesting that exosomes could be a vehicle for the immunosuppressive effects of LMP1 during EBV infection. This receptor is known to negatively regulate both macrophage and T cell activation (54). Additionally, EBV encodes a large number of miRs (55-57) that not only modify the transcriptome of the infected cells, but also that of uninfected cells via exosomes. Pegtel and colleagues demonstrated that EBV-infected B cells release exosomes containing EBV miRs that induce miRmediated repression of EBV target genes such as CXCL11 (58). The ability of EBV miRs to be transferred from infected B cells to noninfected $\mathrm{T}$ cells and monocytes suggests that exosomal transport of viral miRs could contribute to EBV persistence in humans.

The contributions of exosomes to immune evasion and pathogenesis of parasitic organisms, such as Leishmania species pluralis (spp.), have also been well studied. Initial work by Reiner and colleagues established that pathogen-derived exosomes are a vehicle for Leishmania protein secretion and uptake by target macrophages (59), thereby suppressing the immune response (8). A similar effect was seen in vivo, as mice treated with L. major- and L. donovanireleased exosomes prior to infection had a higher parasite load compared with that detected in untreated mice. Proteomic analysis of exosomes from L. mexicana-infected macrophages identified the virulence factor GP63, which could regulate gene expression in recipient cells, leading to a diminished response to infection (60). Moreover, exosomes containing GP63 released from $L$. donovani-infected macrophages target the pre-miR processor dicer1 in hepatocytes to prevent $\mathrm{miR} /$ ribonucleoprotein complex (miRNP) formation, thereby blocking production of miR122, which results in altered serum cholesterol concentration and higher parasite burden (61). Host-derived microvesicles released from cells infected with other parasitic pathogens such as Plasmodium falciparum may also limit host immune surveillance, leading to increased parasite levels or increased pathology (62-64).

There are limited data on exosome-mediated immune suppression in the context of bacterial infections. Nevertheless, studies with mycobacterial components present on or in exosomes have been shown to suppress the immune response, as exosomes from $M$. tuberculosis-infected cells could partially suppress the ability of recipient macrophages to respond to IFN- $\gamma$ (65). This inhibition was dependent on macrophage expression of TLR2 and MyD88 and reflects established mechanisms for TLR2-mediated inhibition of macrophage APC function (66). Moreover, ExMVs released from $M$. tuberculosis-infected macrophages contain lipoarabinomannan (LAM), which inhibits T cell receptor (TCR) signaling and $\mathrm{T}$ cell responses $(67,68)$, suggesting another possible mechanism for vesicle-mediated inhibition of immune responses in this infection model. Exosomes released from cells infected with Mycoplasma induce a mixed cytokine response, including production of both IFN- $\gamma$ and IL-10 from B cells. However, in the context of T cell activation, these exosomes appear to be primarily inhibitory (69). Exosomes released from epithelial cells following Neisseria gonorrhoeae infection contain elevated levels of cellular inhibitor of apoptosis 2 (cIAP2), which may suppress apoptosis of epithelial cells and limit IL-1 $\beta$ production (70). Exosomes can also carry toxins, as shown by Abrami and colleagues, who found that 


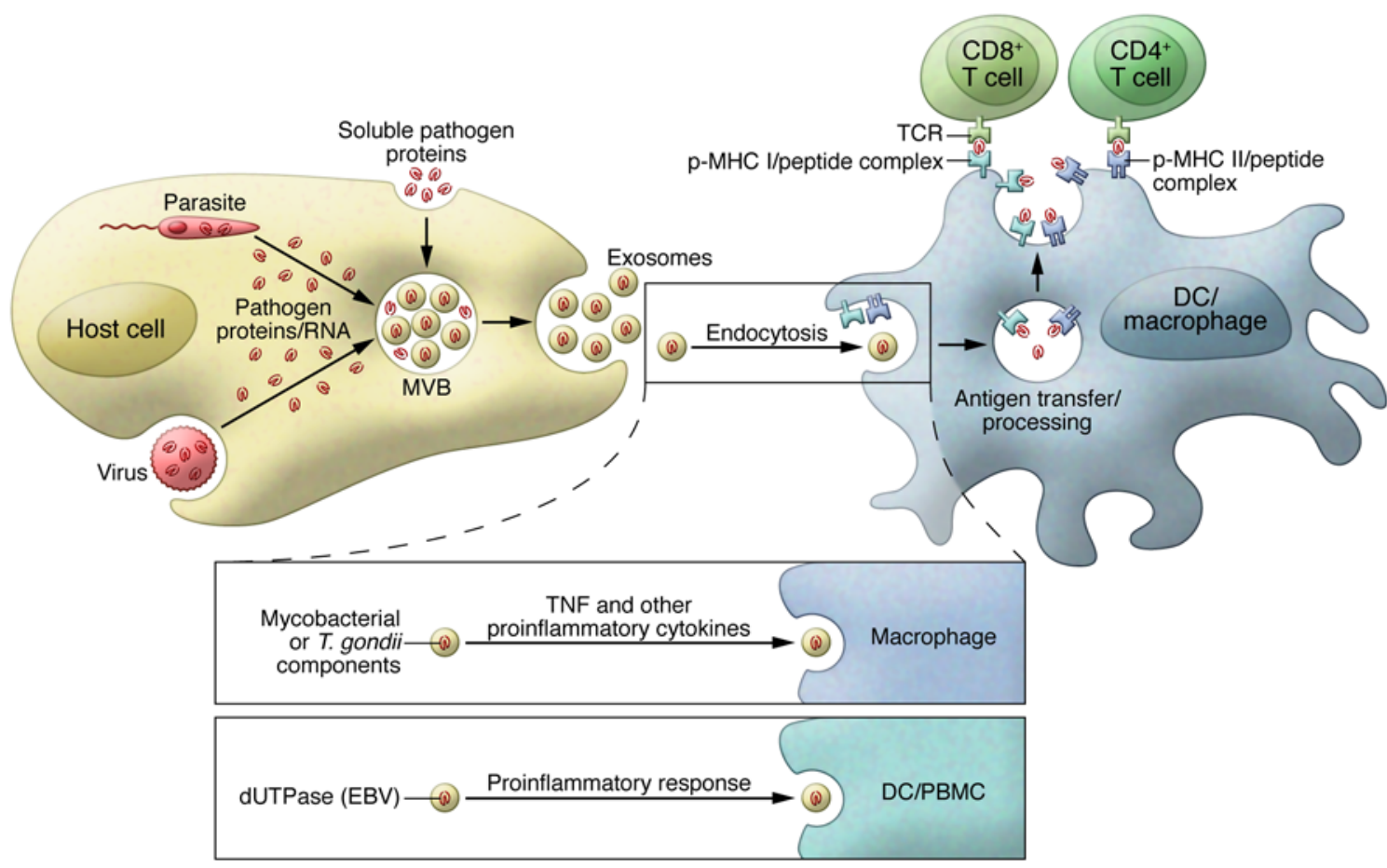

Figure 2. Promoting an effective immune response to pathogen infection through exosomes and other ExMVs. Exosomes and other ExMVs released from virus-, parasite-, and bacteria-infected cells can function through antigen cross priming to activate antigen-specific CD4 ${ }^{+}$and $C D 8^{+} T$ cells. Exosomes from M. tuberculosis- and T. gondii-infected cells also contain PAMPS that can stimulate macrophage production of proinflammatory mediators like TNF- $\alpha$. Similar results were seen with exosomes containing dUTPase released from EBV-infected cells.

lethal factor (LF) produced by Bacillus anthracis was packaged into intraluminal vesicles and released on exosomes when expressed in a human epithelial cell line (71). Several cytotoxic and secreted proteins were also associated with host vesicles released from Chlamydia trachomatis-infected cells, which might function in the delivery of virulence factors (72).

In addition to these prototypical infectious agents, prion diseases, which are spread by an infectious misfolded prion protein (PrPSc), have also been linked to exosomes. Initial studies by Fevrier et al. demonstrated that PrPSc can be transported from infected to uninfected neurons by exosomes (73). The spread of PrPSc by exosomes was not limited to neurons, as non-neuronal cells could also receive the prion protein (74). Recent studies suggest that in addition to carrying PrPSc, the exosomes released from infected neuronal cells have a different repertoire of miRs compared with that of exosomes released from uninfected cells (75). This includes miRs that are associated with neurological disorders such as miR128a and miR146a. The importance of exosomes in prion transmission from cell to cell or organism to organism is still being defined.

Contributions of ExMVs to host defense and immune responses. Exosomes can function as antigen carriers, promoting an acquired immune response (Figure 2). T cell activation can be mediated by exosomes directly, as they have been shown to carry processed antigen as well as MHC class I and II molecules and costimulatory molecules (76-78); however, direct activation may be limited (79-81), and the major mechanism of T cell activation by exosomes is likely through their uptake by APCs and subsequent presentation by host cell MHC molecules $(82,83)$. Independently of the mech- anism of $\mathrm{T}$ cell activation, exosomes, such as those released from CMV-infected human endothelial cells, can stimulate memory $\mathrm{CD}^{+} \mathrm{T}$ cells isolated from CMV-infected donors (84). Considering the overlap in the host components involved in virus and exosome biosynthesis and the presence of viral proteins in exosomes, it is likely that viral infections generally produce exosomes that can stimulate a $\mathrm{T}$ cell response; however, this has only been directly evaluated for a limited number of viral infections (85). Exosomemediated antigen delivery is not limited to viral infections, as studies by Aline and colleagues found that DCs pulsed with Toxoplasma gondii proteins released exosomes that could stimulate a protective immune response against acute and chronic T. gondii infection when the DCs were adoptively transferred to mice. This response was antigen specific and included both cellular and humoral immunity (86). Martin-Jaular and colleagues found that exosomes isolated from the blood of Plasmodium yoelii-infected $\mathrm{BALB} / \mathrm{c}$ mice contained parasite proteins and, when used as a vaccine in naive mice, provided protection against subsequent $P$. yoelii infection (87). These exosomes stimulated the production of IgG Abs that recognized P. yoelii-infected rbc, decreased the level of parasitemia, and improved survival. Exosomes released from $M$. tuberculosis- or bacillus Calmette-Guérin-infected (BCG-infected) cells or from M. tuberculosis culture filtrate protein-treated (CFPtreated) macrophages were shown to activate antigen-specific $\mathrm{CD}^{+}$and $\mathrm{CD}^{+} \mathrm{T}$ cells in vivo and promote the activation and maturation of BM-derived DCs (BMDCs) (88). Moreover, vaccination of mice with exosomes released from CFP-treated macrophages protects against a low-dose, aerosolized $M$. tuberculosis inoculation (89). The release of mycobacterial antigens from infected macro- 
phages can be exosome independent, as studies by Ramachandra et al. found that infection with M. tuberculosis or BCG resulted in increased release of both exosomes and microvesicles, which stimulated an antigen-specific $\mathrm{T}$ cell response (79). Bacterial membrane vesicles (BMVs) derived directly from intracellular $M$. tuberculosis are released from host cells $(2,10)$ and may potentially contribute to $\mathrm{T}$ cell responses.

The beneficial properties of exosomes are not restricted to their ability to carry antigens. During an HIV-1 infection, exosomes released from $\mathrm{CD} 8^{+} \mathrm{T}$ cells can suppress HIV-1 transcription within infected cells in a protein-dependent, but antigen-independent, manner (90). Furthermore, host-derived exosomes containing the cytidine deaminase APOBEC3G can inhibit HIV replication in the recipient cells (91). Exosomes from EBV-infected cells have also been shown to contain dUTPase and can induce a proinflammatory/antiviral response through activation of NF- $\kappa \mathrm{B}$ and production of cytokines (92). Dreux and colleagues reported that exosomes released from $\mathrm{HCV}$-infected cells can induce IFN- $\alpha$ production from uninfected plasmacytoid DCs and that this activity is dependent on viral RNA present within the exosomes (40). These results suggest that export of viral RNA may serve both as a viral strategy to evade pathogen sensing within infected cells and as a host strategy to induce an innate response in bystander cells. Similar findings were reported by $\mathrm{Li}$ and colleagues, who showed that IFN- $\alpha$-treated liver nonparenchymal cells release exosomes that contain a number of host molecules with antiviral activity (93). These and other studies suggest that infected cell-derived exosomes and other ExMVs can promote the innate and acquired immune response through cytokine production $(93,94)$. However, additional studies are needed to unravel how and when these ExMVs promote or limit antiviral immunity. One of the difficulties in this type of analysis is the separation of viral particles from exosomes, as they are often of similar size and density (19).

Exosomes can also promote effective immune responses to nonviral pathogens. Our previous studies indicate that exosomes released from THP-1 cells after T. gondii infection stimulate noninfected THP- 1 cells to produce TNF- $\alpha$ and other proinflammatory mediators (95), although the exosomal component responsible for this activity remains undefined. Infection with the gastrointestinal parasite Cryptosporidium parvum was shown to increase exosome release from intestinal and biliary epithelial cells into the lumen of the gastrointestinal tract (46). These exosomes also carried antimicrobial peptides from the epithelial cells, the export of which was increased through TLR 4 activation by the parasite. Couper and colleagues demonstrated that microvesicles isolated from the plasma of malaria-infected, but not naive, mice induce potent, TLR4-dependent activation of macrophages in vitro, as measured by CD40 upregulation and TNF- $\alpha$ production (64). Exosomes released from Mycobacterium avium-infected macrophages stimulated a proinflammatory response in noninfected or "bystander" macrophages in a TLR- and MyD88-dependent manner (96). Anand and colleagues observed increased exosome production in macrophages infected with M. avium and Mycobacterium smegmatis compared with that detected in uninfected cells, as well as increased levels of the host protein HSP70, which activates macrophages in vitro (97). Importantly, exosomes released from M. tuberculosis- or BCG-infected macrophages were also shown to be proinflammatory (95), and exosomes isolated from the bronchoalveolar lavage fluid (BALF) of BCG-infected mice contained mycobacterial components and were proinflammatory ex vivo (95). Moreover, the presence of TLR2-agonist lipoproteins in exosomes from $M$. tuberculosis-infected cells may allow TLR2-mediated enhancement of $\mathrm{T}$ cell responses, which has been reported for $M$. tuberculosis lipoproteins $(98,99)$. Together, these results suggest that exosomes from mycobacteria-infected cells can promote both the recruitment and activation of immune cells and may play a role in promoting the innate and acquired immune response upon mycobacterial infection.

\section{Use of ExMVs in therapeutics}

Soon after ExMVs were found to carry tumor antigens and stimulate an antitumor response in mice $(100,101)$, attention turned to their potential use as antitumor vaccines in humans, and clinical trials are ongoing to evaluate this possible clinical application $(102,103)$. The interest in using exosomes and other ExMVs as vaccines in disease diagnostics and as drug carriers has increased dramatically over the past few years. In the section below, we highlight some of the infectious diseases studies that evaluated ExMVs as vaccines, disease markers, and drug carriers, as well as some of the advantages and caveats associated with their use.

Use of ExMVs as vaccines against pathogens. There are a number of potential advantages to using exosomes as vaccines against pathogens, including (a) improved distribution of antigens due to the ability of exosomes to circulate in bodily fluids and reach distal organs; (b) stable conditions for maintaining protein structure; (c) efficient binding and uptake by APCs due to the expression of adhesion molecules on exosomes; and (d) exosomes as the body's natural means for transferring antigens between cells. Although exosomes have a number of advantages over conventional vaccine strategies, they have been evaluated as vaccines against viral pathogens only sparingly and with limited demonstration of their efficacy $(85,104)$. In contrast, a number of studies have evaluated exosomes as vaccines against other types of pathogens. For example, Schnitzer and colleagues showed that DC-derived exosomes containing Leishmania antigens provide protective immunity against cutaneous leishmaniasis when used as a vaccine (105). Colino and colleagues isolated exosomes from BMDCs treated with diptheria toxin (DT) and found that the exosomes stimulated an IgG response specific for DT (106). Similar results were obtained with Streptococcus pneumoniae. Exosomes released from BMDCs pulsed with $S$. pneumoniae capsular polysaccharide 14 (Cps14) antigen could stimulate protective IgM and $\operatorname{IgG}$ responses against $S$. pneumoniae infection (107).

Although exosomes could potentially be used as cell-free vaccines, there are both practical and conceptual issues that need to be addressed prior to their use in humans. These include producing exosomes with the correct antigen composition, developing methods to reproducibly generate exosomes with the proper content, and the risks of introducing nonself human molecules into vaccinated individuals. This latter point is particularly important, as the exosomes used for large-scale vaccination will likely be produced from a human cell line and therefore will have proteins and other molecules specific to this cell line. The effect of these foreign antigens on the recipient's immune response is unclear, and 
additional studies are needed to delineate potential risks. Nevertheless, exosomes provide a unique approach to vaccine development, and this area of investigation will likely continue to receive significant attention in the coming years.

ExMVs as diagnostic markers for infectious diseases. A number of studies have shown quantitative and qualitative differences in exosome composition between healthy and diseased individuals (reviewed in ref. 108). These changes in exosome composition, combined with their easy accessibility, make exosomes excellent biomarker candidates. Moreover, exosomes can be an enriched source of pathogen and host protein and RNA. Many sources for disease biomarker detection (e.g., serum or plasma) are very complex, and most of the components are not valuable in the context of biomarkers. This leads to a high background and more difficult detection of the targeted proteins and RNA. Using purified exosomes greatly diminishes this background. Nevertheless, the use of exosomes in the diagnosis of infectious diseases is relatively new, but shows great promise, as the markers can be both hostand pathogen-derived. In support of their potential as biomarkers, we found the exosome concentration in serum from BCG- or M. tuberculosis-infected mice to be significantly higher when compared with serum exosomes from uninfected controls, and this concentration correlated with bacterial load (45). Moreover, exosomes isolated from $M$. tuberculosis-infected mice or the serum of tuberculosis (TB) patients contained mycobacterial proteins, suggesting that exosomes could be used as markers of active disease $(109,110)$. Surprisingly, we have also found that M. tuberculosisinfected macrophages release exosomes containing mycobacterial RNA (43), hinting at the possibility that exosomal RNA could also be a useful marker for active TB. Markers are not limited to pathogen-derived molecules, as host components may also differ qualitatively and quantitatively between exosomes from an infected individual and those from uninfected individuals, as has been shown for CD81 in serum ExMVs from patients with chronic hepatitis C (111). Nevertheless, the use of host components is more problematic, as these factors may be less specific and shared among a number of diseases (e.g. inflammatory mediators).

ExMVs and delivery of therapeutic molecules. The observation that exosomes can deliver RNA to recipient host cells (35) has generated great interest in the use of exosomes in drug delivery applications. The most attention has been paid to the delivery of RNA (siRNA, miR), but studies have also suggested the utility of exosomes for the delivery of chemotherapeutic agents for cancer and inhibitors of specific enzymes. Most studies of exosomes for drug delivery have used cancer models, and their potential for treatment of infectious diseases is less clear. It is possible that exosomes would have advantages for the treatment of intracellular infections if exosomes could be engineered to target drugs to the infected cells. Additionally, the study of exosomes may reveal functions and mechanisms that could be incorporated into the design of artificial vesicle nanoparticles for drug delivery and other therapeutic applications.

\section{Conclusion and future directions}

Although our knowledge of how exosomes and other ExMVs function in the context of infectious diseases is still limited, the data published thus far have generated interesting and compelling information. Continued growth in this area should lead to a better understanding of virulence mechanisms and immune responses as well as the development of new diagnostics and vaccines. This growth will require developing a better understanding of the cell types that produce ExMVs and the composition of these ExMVs. Defining exosome composition, both in the context of host and microbial components and how this changes during the course of an infection, is critical to defining the functional role of exosomes. Other questions include defining the ExMV recipient cells, the cellular response to exosome engagement, and the interplay between exosome-mediated responses and other components of an immune response. However, to be successful, we to need to develop new methods, including the ability to specifically block the production of exosomes and other ExMVs, coupled with approaches for the evaluation of disease outcome. These approaches will help us understand whether exosomes function to benefit the host, or are used by pathogens to subvert the immune response. It is likely that both of these mechanisms exist and that the outcome is determined by a balance of drivers of immune responses and immune evasion to which ExMVs contribute. Further studies are needed to define this balance and determine how we can manipulate the process to our benefit.

Address correspondence to: Jeffrey S. Schorey, Department of Biological Sciences, University of Notre Dame, 129 Galvin Life Science Center, Notre Dame, Indiana 46556, USA. Phone: 574.631.3734.E-mail: schorey.1@nd.edu.
1. Prados-Rosales R, et al. Mycobacterial membrane vesicles administered systemically in mice induce a protective immune response to surface compartments of Mycobacterium tuberculosis. MBio. 2014;5(5):e01921-14.

2. Prados-Rosales R, et al. Mycobacteria release active membrane vesicles that modulate immune responses in a TLR2-dependent manner in mice. J Clin Invest. 2011;121(4):1471-1483.

3. Schwechheimer C, Kuehn MJ. Outer-membrane vesicles from Gram-negative bacteria: biogenesis and functions. Nat Rev Microbiol. 2015;13(10):605-619.

4. Brown L, Wolf JM, Prados-Rosales R, Casadevall A. Through the wall: extracellular vesicles in Gram-positive bacteria, mycobacteria and fungi.
Nat Rev Microbiol. 2015;13(10):620-630.

5. Acevedo R, et al. Bacterial outer membrane vesicles and vaccine applications. Front Immunol. 2014;5:121.

6. Eisenman HC, Frases S, Nicola AM, Rodrigues ML, Casadevall A. Vesicle-associated melanization in Cryptococcus neoformans. Microbiology. 2009;155(pt 12):3860-3867.

7. Oliveira DL, Freire-de-Lima CG, Nosanchuk JD, Casadevall A, Rodrigues ML, Nimrichter L. Extracellular vesicles from Cryptococcus neoformans modulate macrophage functions. Infect Immun. 2010;78(4):1601-1609.

8. Silverman JM, et al. Leishmania exosomes modulate innate and adaptive immune responses through effects on monocytes and dendritic cells.
Jimmunol. 2010;185(9):5011-5022.

9. D'Souza-Schorey C, Clancy JW. Tumor-derived microvesicles: shedding light on novel microenvironment modulators and prospective cancer biomarkers. Genes Dev. 2012;26(12):1287-1299.

10. Athman JJ, Wang Y, McDonald DJ, Boom WH, Harding CV, Wearsch PA. Bacterial membrane vesicles mediate the release of mycobacterium tuberculosis lipoglycans and lipoproteins from infected macrophages. JImmunol. 2015;195(3):1044-1053.

11. Beatty WL, Russell DG. Identification of mycobacterial surface proteins released into subcellular compartments of infected macrophages. Infect Immun. 2000;68(12):6997-7002.

12. Beatty WL, Ullrich HJ, Russell DG. Mycobacterial 
surface moieties are released from infected macrophages by a constitutive exocytic event. Eur J Cell Biol. 2001;80(1):31-40.

13. Wolf JM, Davis DA. Mutational analysis of Candida albicans SNF7 reveals genetically separable Rim101 and ESCRT functions and demonstrates divergence in bro1-domain protein interactions. Genetics. 2010;184(3):673-694.

14. Pan BT, Johnstone RM. Fate of the transferrin receptor during maturation of sheep reticulocytes in vitro: selective externalization of the receptor. Cell.1983;33(3):967-978.

15. Pan BT, Blostein R, Johnstone RM. Loss of the transferrin receptor during the maturation of sheep reticulocytes in vitro. Biochem $\mathrm{J}$. 1983;210(1):37-47.

16. Hanson PI, Cashikar A. Multivesicular body morphogenesis. Annu Rev Cell Dev Biol. 2012;28:337-362.

17. Tamai $\mathrm{K}$, et al. Exosome secretion of dendritic cells is regulated by Hrs, an ESCRT- 0 protein. Biochem Biophys Res Commun. 2010;399(3):384-390.

18. Hurley JH. ESCRTs are everywhere. EMBO J. 2015;34(19):2398-2407.

19. Meckes DG. Exosomal communication goes viral. J Virol. 2015;89(10):5200-5203.

20. Gambarte Tudela J, et al. The late endocytic Rab39a GTPase regulates the interaction between multivesicular bodies and chlamydial inclusions. J Cell Sci. 2015;128(16):3068-3081.

21. Boonyaratanakornkit J, Schomacker H, Collins P, Schmidt A. Alix serves as an adaptor that allows human parainfluenza virus type 1 to interact with the host cell ESCRT system. PLoS One. 2013;8(3):e59462.

22. Mehra A, et al. Mycobacterium tuberculosis type VII secreted effector EsxH targets host ESCRT to impair trafficking. PLoS Pathog. 2013;9(10):e1003734.

23. Sreekumar PG, et al. $\alpha \mathrm{B}$ crystallin is apically secreted within exosomes by polarized human retinal pigment epithelium and provides neuroprotection to adjacent cells. PLoS One. 2010;5(10):e12578.

24. Raposo G, Stoorvogel W. Extracellular vesicles: exosomes, microvesicles, and friends. J Cell Biol. 2013;200(4):373-383.

25. Andreu Z, Yanez-Mo M. Tetraspanins in extracellular vesicle formation and function. Front Immunol. 2014;5:442.

26. Zhang HG, Grizzle WE. Exosomes: a novel pathway of local and distant intercellular communication that facilitates the growth and metastasis of neoplastic lesions. Am J Pathol. 2014;184(1):28-41.

27. Subra C, Laulagnier K, Perret B, Record M. Exosome lipidomics unravels lipid sorting at the level of multivesicular bodies. Biochimie. 2007;89(2):205-212.

28. Choi DS, Kim DK, Kim YK, Gho YS. Proteomics, transcriptomics and lipidomics of exosomes and ectosomes. Proteomics. 2013;13(10-11):1554-1571.

29. Wood SL, Knowles MA, Thompson D, Selby PJ, Banks RE. Proteomic studies of urinary biomarkers for prostate, bladder and kidney cancers. Nat Rev Urol. 2013;10(4):206-218.

30. Lenassi M, et al. HIV Nef is secreted in exosomes and triggers apoptosis in bystander $\mathrm{CD} 4^{+} \mathrm{T}$ cells.
Traffic. 2010;11(1):110-122.

31. Campbell TD, Khan M, Huang MB, Bond VC, Powell MD. HIV-1 Nef protein is secreted into vesicles that can fuse with target cells and virions. Ethn Dis. 2008;18(2 suppl 2):S2-S14.

32. Giri PK, Kruh NA, Dobos KM, Schorey JS. Proteomic analysis identifies highly antigenic proteins in exosomes from M. Proteomics. 2010;10(17):3190-3202.

33. Smith VL, Jackson L, Schorey JS. Ubiquitination as a mechanism to transport soluble mycobacterial and eukaryotic proteins to exosomes. JImmunol. 2015;195(6):2722-2730.

34. Ratajczak J, et al. Embryonic stem cell-derived microvesicles reprogram hematopoietic progenitors: evidence for horizontal transfer of mRNA and protein delivery. Leukemia. 2006;20(5):847-856.

35. Valadi H, Ekstrom K, Bossios A, Sjostrand M, Lee JJ, Lotvall JO. Exosome-mediated transfer of mRNAs and microRNAs is a novel mechanism of genetic exchange between cells. Nat Cell Biol. 2007;9(6):654-659.

36. Lee Y, El Andaloussi S, Wood MJ. Exosomes and microvesicles: extracellular vesicles for genetic information transfer and gene therapy. Hum Mol Genet. 2012;21(R1):R125-R134.

37. Eldh M, et al. Exosomes communicate protective messages during oxidative stress; possible role of exosomal shuttle RNA. PLoS One. 2010;5(12):e15353.

38. Mittelbrunn M, et al. Unidirectional transfer of microRNA-loaded exosomes from $\mathrm{T}$ cells to antigen-presenting cells. Nat Commun. 2011;2:282.

39. Montecalvo A, et al. Mechanism of transfer of functional microRNAs between mouse dendritic cells via exosomes. Blood. 2012;119(3):756-766.

40. Dreux M, et al. Short-range exosomal transfer of viral RNA from infected cells to plasmacytoid dendritic cells triggers innate immunity. Cell Host Microbe. 2012;12(4):558-570.

41. Ghoujal B, Milev MP, Ajamian L, Abel K, Mouland AJ. ESCRT-II's involvement in HIV-1 genomic RNA trafficking and assembly. Biol Cell. 2012;104(12):706-721.

42. Columba Cabezas S, Federico M. Sequences within RNA coding for HIV-1 Gag p17 are efficiently targeted to exosomes. Cell Microbiol. 2013;15(3):412-429.

43. Singh PP, Li L, Schorey JS. Exosomal RNA from Mycobacterium tuberculosis-infected cells is functional in recipient macrophages. Traffic. 2015;16(6):555-571.

44. Nantakomol D, et al. Quantitation of cell-derived microparticles in plasma using flow rate based calibration. Southeast Asian J Trop Med Public Health. 2008;39(1):146-153.

45. Singh PP, Smith VL, Karakousis PC, Schorey JS. Exosomes isolated from mycobacteria-infected mice or cultured macrophages can recruit and activate immune cells in vitro and in vivo. J Immunol. 2012;189(2):777-785.

46. Hu G, et al. Release of luminal exosomes contributes to TLR4-mediated epithelial antimicrobial defense. PLoS Pathog. 2013;9(4):e1003261.

47. Plazolles N, Humbert JM, Vachot L, Verrier B, Hocke C, Halary F. Pivotal advance: the promotion of soluble DC-SIGN release by inflammatory signals and its enhancement of cytomegalovirus- mediated cis-infection of myeloid dendritic cells. JLeukoc Biol. 2011;89(3):329-342.

48. Masciopinto F, et al. Association of hepatitis C virus envelope proteins with exosomes. Eur J Immunol. 2004;34(10):2834-2842.

49. Mack M, et al. Transfer of the chemokine receptor CCR 5 between cells by membrane-derived microparticles: a mechanism for cellular human immunodeficiency virus 1 infection. Nat Med. 2000;6(7):769-775.

50. Rozmyslowicz T, et al. Platelet- and megakaryocyte-derived microparticles transfer CXCR4 receptor to CXCR4-null cells and make them susceptible to infection by X4-HIV. AIDS. 2003;17(1):33-42.

51. Wang T, et al. Transfer of intracellular HIV Nef to endothelium causes endothelial dysfunction. PLoS One. 2014;9(3):e91063.

52. Klibi J, et al. Blood diffusion and Th1-suppressive effects of galectin-9-containing exosomes released by Epstein-Barr virus-infected nasopharyngeal carcinoma cells. Blood. 2009;113(9):1957-1966.

53. Dukers DF, et al. Direct immunosuppressive effects of EBV-encoded latent membrane protein 1. JImmunol. 2000;165(2):663-670.

54. Ikeda O, et al. STAP-2 negatively regulates both canonical and noncanonical NF-kappaB activation induced by Epstein-Barr virusderived latent membrane protein 1. Mol Cell Biol. 2008;28(16):5027-5042.

55. Yang HJ, et al. Comprehensive profiling of Epstein-Barr virus-encoded miRNA species associated with specific latency types in tumor cells. Virol J. 2013;10:314.

56. Sullivan CS, Grundhoff AT, Tevethia S, Pipas JM, Ganem D. SV40-encoded microRNAs regulate viral gene expression and reduce susceptibility to cytotoxic T cells. Nature. 2005;435(7042):682-686.

57. Pfeffer S, et al. Identification of virus-encoded microRNAs. Science. 2004;304(5671):734-736.

58. Pegtel DM, et al. Functional delivery of viral miRNAs via exosomes. Proc Natl Acad Sci U S A. 2010;107(14):6328-6333.

59. Silverman JM, et al. An exosome-based secretion pathway is responsible for protein export from Leishmania and communication with macrophages. JCell Sci. 2010;123(pt 6):842-852.

60. Hassani K, Olivier M. Immunomodulatory impact of leishmania-induced macrophage exosomes: a comparative proteomic and functional analysis. PLoS Negl Trop Dis. 2013;7(5):e2185.

61. Ghosh J, Bose M, Roy S, Bhattacharyya SN. Leishmania donovani targets Dicer1 to downregulate miR-122, lower serum cholesterol, and facilitate murine liver infection. Cell Host Microbe. 2013;13(3):277-288.

62. Mantel PY, Marti M. The role of extracellular vesicles in Plasmodium and other protozoan parasites. Cell Microbiol. 2014;16(3):344-354.

63. Barteneva NS, Maltsev N, Vorobjev IA. Microvesicles and intercellular communication in the context of parasitism. Front Cell Infect Microbiol. 2013;3:49.

64. Couper KN, et al. Parasite-derived plasma microparticles contribute significantly to malaria infection-induced inflammation through 
potent macrophage stimulation. PLoS Pathog. 2010;6(1):e1000744.

65. Singh PP, LeMaire C, Tan JC, Zeng E, Schorey JS. Exosomes released from M. PLoS One. 2011;6(4):e18564.

66. Harding CV, Boom WH. Regulation of antigen presentation by Mycobacterium tuberculosis: a role for Toll-like receptors. Nat Rev Microbiol. 2010;8(4):296-307.

67. Mahon RN, Sande OJ, Rojas RE, Levine AD, Harding CV, Boom WH. Mycobacterium tuberculosis ManLAM inhibits T-cell-receptor signaling by interference with ZAP-70, Lck and LAT phosphorylation. Cell Immunol. 2012;275(1-2):98-105.

68. Yang C, Ruffner MA, Kim SH, Robbins PD. Plasma-derived MHC class II+ exosomes from tumor-bearing mice suppress tumor antigen-specific immune responses. Eur J Immunol. 2012;42(7):1778-1784.

69. Yang C, Chalasani G, Ng YH, Robbins PD. Exosomes released from Mycoplasma infected tumor cells activate inhibitory B cells. PLoS One. 2012;7(4):e36138.

70. Nudel K, Massari P, Genco CA. Neisseria gonorrhoeae modulates cell death in human endocervical epithelial cells through export of exosome-associated cIAP2. Infect Immun. 2015;83(9):3410-3417.

71. Abrami L, et al. Hijacking multivesicular bodies enables long-term and exosome-mediated long-distance action of anthrax toxin. Cell Rep. 2013;5(4):986-996.

72. Ettelaie C, Collier ME, James NJ, Li C. Induction of tissue factor expression and release as microparticles in ECV304 cell line by Chlamydia pneumoniae infection. Atherosclerosis. 2007;190(2):343-351.

73. Fevrier B, et al. Cells release prions in association with exosomes. Proc Natl Acad Sci U S A. 2004;101(26):9683-9688.

74. Vella LJ, Sharples RA, Lawson VA, Masters CL, Cappai R, Hill AF. Packaging of prions into exosomes is associated with a novel pathway of PrP processing. J Pathol. 2007;211(5):582-590.

75. Bellingham SA, Coleman BM, Hill AF. Small RNA deep sequencing reveals a distinct miRNA signature released in exosomes from prion-infected neuronal cells. Nucleic Acids Res. 2012;40(21):10937-10949.

76. Utsugi-Kobukai S, Fujimaki H, Hotta C, Nakazawa M, Minami M. MHC class I-mediated exogenous antigen presentation by exosomes secreted from immature and mature bone marrow derived dendritic cells. Immunol Lett. 2003;89(2-3):125-131.

77. Admyre C, Johansson SM, Paulie S, Gabrielsson S. Direct exosome stimulation of peripheral human T cells detected by ELISPOT. Eur J Immunol. 2006;36(7):1772-1781.

78. Luketic L, et al. Antigen presentation by exosomes released from peptide-pulsed dendritic cells is not suppressed by the presence of active CTL. JImmunol. 2007;179(8):5024-5032.

79. Ramachandra L, et al. Mycobacterium tuberculosis synergizes with ATP to induce release of microvesicles and exosomes containing major histocompatibility complex class II molecules capable of antigen presentation. Infect Immun.
2010;78(12):5116-5125.

80. Raposo G, et al. B lymphocytes secrete antigen-presenting vesicles. J Exp Med 1996;183(3):1161-1172.

81. Vincent-Schneider H, et al. Exosomes bearing HLA-DR1 molecules need dendritic cells to efficiently stimulate specific T cells. Int Immunol. 2002;14(7):713-722.

82. Montecalvo A, et al. Exosomes as a short-range mechanism to spread alloantigen between dendritic cells during T cell allorecognition. J Immunol. 2008;180(5):3081-3090.

83. Qazi KR, Gehrmann U, Domange Jordo E, Karlsson MC, Gabrielsson S. Antigen-loaded exosomes alone induce Th1-type memory through a B-cell-dependent mechanism. Blood. 2009;113(12):2673-2683.

84. Walker JD, Maier CL, Pober JS. Cytomegalovirus-infected human endothelial cells can stimulate allogeneic $\mathrm{CD} 4^{+}$memory $\mathrm{T}$ cells by releasing antigenic exosomes. Jimmunol. 2009;182(3):1548-1559.

85. Kuate S, Cinatl J, Doerr HW, Uberla K. Exosomal vaccines containing the $S$ protein of the SARS coronavirus induce high levels of neutralizing antibodies. Virology. 2007;362(1):26-37.

86. Aline F, Bout D, Amigorena S, Roingeard P, Dimier-Poisson I. Toxoplasma gondii antigenpulsed-dendritic cell-derived exosomes induce a protective immune response against T. Infect Immun. 2004;72(7):4127-4137.

87. Martin-Jaular L, Nakayasu ES, Ferrer M, Almeida IC, Del Portillo HA. Exosomes from Plasmodium yoelii-infected reticulocytes protect mice from lethal infections. PLoS One. 2011;6(10):e26588.

88. Giri PK, Schorey JS. Exosomes derived from M. PLoS One. 2008;3(6):e2461.

89. Cheng Y, Schorey JS. Exosomes carrying mycobacterial antigens can protect mice against Mycobacterium tuberculosis infection. Eur J Immunol. 2013;43(12):3279-3290.

90. Tumne A, et al. Noncytotoxic suppression of human immunodeficiency virus type 1 transcription by exosomes secreted from $\mathrm{CD}^{+} \mathrm{T}$ cells. J Virol. 2009;83(9):4354-4364.

91. Khatua AK, Taylor HE, Hildreth JE, Popik W. Exosomes packaging APOBEC3G confer human immunodeficiency virus resistance to recipient cells. J Virol. 2009;83(2):512-521.

92. Ariza ME, Rivailler P, Glaser R, Chen M, Williams MV. Epstein-Barr virus encoded dUTPase containing exosomes modulate innate and adaptive immune responses in human dendritic cells and peripheral blood mononuclear cells. PLOS One. 2013;8(7):e69827.

93. Li J, et al. Exosomes mediate the cell-to-cell transmission of IFN- $\alpha$-induced antiviral activity. Nat Immunol. 2013;14(8):793-803.

94. Wakim LM, Bevan MJ. Cross-dressed dendritic cells drive memory CD $8^{+} \mathrm{T}$-cell activation after viral infection. Nature. 2011;471(7340):629-632.

95. Bhatnagar S, Shinagawa K, Castellino FJ, Schorey JS. Exosomes released from macrophages infected with intracellular pathogens stimulate a proinflammatory response in vitro and in vivo. Blood. 2007;110(9):3234-3244.

96. Bhatnagar S, Schorey JS. Exosomes released from infected macrophages contain Mycobacterium avium glycopeptidolipids and are proinflammatory. J Biol Chem. 2007;282(35):25779-25789.

97. Anand PK, Anand E, Bleck CK, Anes E, Griffiths G. Exosomal Hsp70 induces a pro-inflammatory response to foreign particles including mycobacteria. PLoS One. 2010;5(4):e10136.

98. Reba SM, et al. TLR2 engagement on CD4(+) T cells enhances effector functions and protective responses to Mycobacterium tuberculosis. Eur J Immunol. 2014;44(5):1410-1421.

99. Lancioni CL, et al. Mycobacterium tuberculosis lipoproteins directly regulate human memory CD4(+) T cell activation via Toll-like receptors 1 and 2. Infect Immun. 2011;79(2):663-673.

100.Zitvogel L, et al. Eradication of established murine tumors using a novel cell-free vaccine: dendritic cell-derived exosomes. Nat Med. 1998;4(5):594-600.

101.Andre F, et al. Exosomes as potent cellfree peptide-based vaccine. J Immunol. 2004;172(4):2126-2136.

102. Pitt JM, et al. Dendritic cell-derived exosomes as immunotherapies in the fight against cancer. J Immunol. 2014;193(3):1006-1011.

103. Chaput N, et al. Dendritic cell derived-exosomes: biology and clinical implementations. J Leukoc Biol. 2006;80(3):471-478.

104.Zhu L, Song H, Zhang X, Xia X, Sun H. Inhibition of porcine reproductive and respiratory syndrome virus infection by recombinant adenovirus- and/or exosome-delivered the artificial microRNAs targeting sialoadhesin and CD163 receptors. Virol J. 2014;11:225.

105. Schnitzer JK, Berzel S, Fajardo-Moser M, Remer KA, Moll H. Fragments of antigen-loaded dendritic cells (DC) and DC-derived exosomes induce protective immunity against Leishmania major. Vaccine. 2010;28(36):5785-5793.

106. Colino J, Snapper CM. Exosomes from bone marrow dendritic cells pulsed with diphtheria toxoid preferentially induce type 1 antigen-specific $\operatorname{IgG}$ responses in naive recipients in the absence of free antigen. J Immunol. 2006;177(6):3757-3762.

107. Colino J, Snapper CM. Dendritic cell-derived exosomes express a Streptococcus pneumoniae capsular polysaccharide type 14 cross-reactive antigen that induces protective immunoglobulin responses against pneumococcal infection in mice. Infect Immun. 2007;75(1):220-230.

108. Chaput N, Thery C. Exosomes: immune properties and potential clinical implementations. Semin Immunopathol. 2011;33(5):419-440.

109.Kruh-Garcia N, Schorey JS, Dobos K. Exosomes: new tuberculosis biomarkers: prospects from the bench to the clinic. In: Cardona PJ, ed. Understanding Tuberculosis-Global Experiences and Innovative Approaches to the Diagnosis. Rijeka, Croatia: InTech - Open Access Publisher; 2011:395-410. http:// www.intechopen.com/download/pdf/28549.

110. Kruh-Garcia NA, et al. Detection of Mycobacterium tuberculosis peptides in the exosomes of patients with active and latent M. tuberculosis infection using MRM-MS. PLoS One. 2014;9(7):e103811.

111. Welker MW, et al. Soluble serum CD81 is elevated in patients with chronic hepatitis $C$ and correlates with alanine aminotransferase serum activity. PLoS One. 2012;7(2):e30796. 